Document downloaded from:

http://hdl.handle.net/10251/117898

This paper must be cited as:

Avella-Oliver, M.; Ferrando Martín, V.; Monsoriu Serra, JA.; Puchades, R.; Maquieira Catala, A. (2018). A label-free diffraction-based sensing displacement immunosensor toquantify low molecular weight organic compounds. Analytica Chimica Acta. 1033:173-179. https://doi.org/10.1016/j.aca.2018.05.060

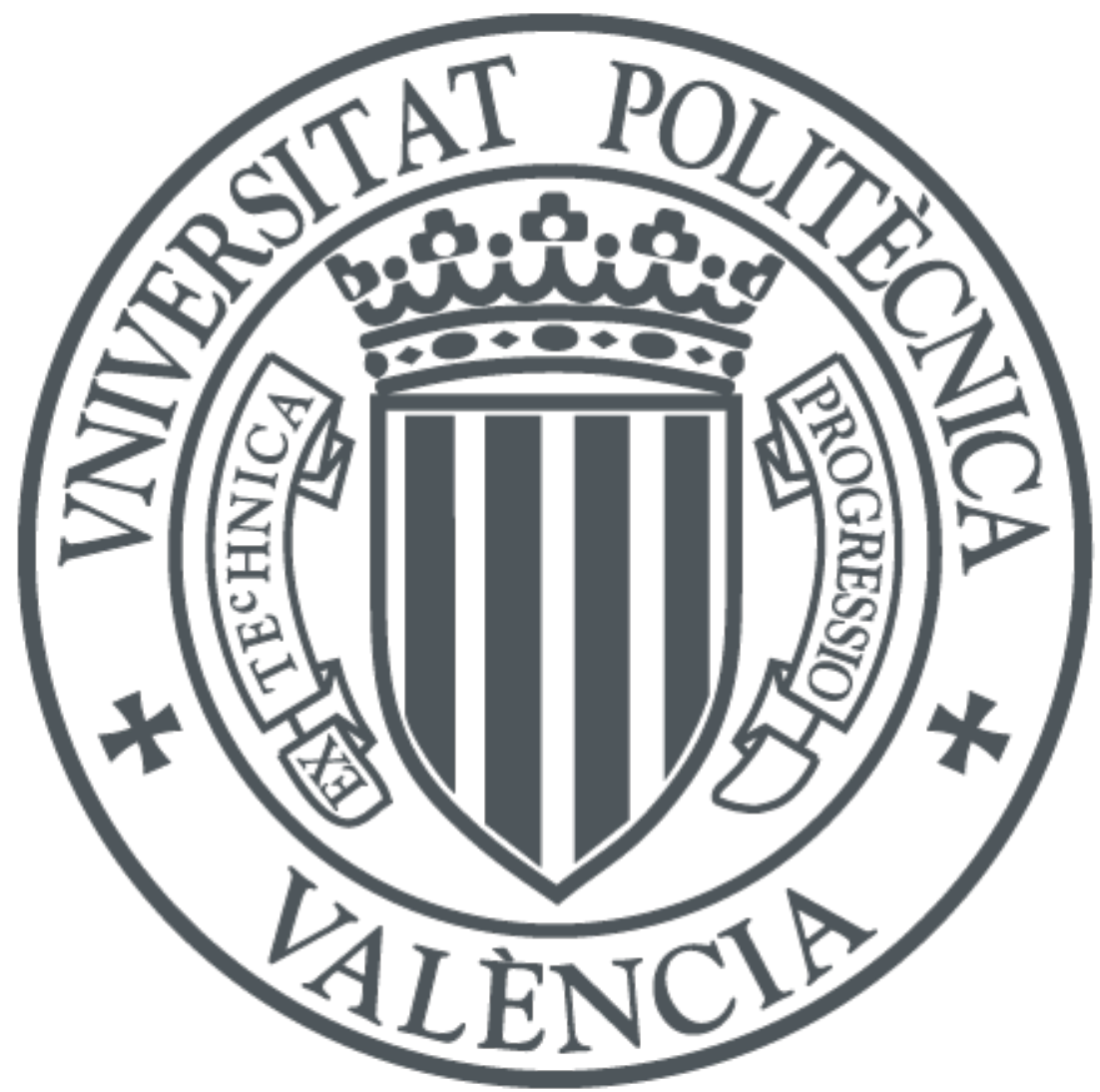

The final publication is available at

http://doi.org/10.1016/j.aca.2018.05.060

Copyright Elsevier

Additional Information 


\title{
A label-free diffraction-based sensing displacement immunosensor to quantify low molecular weight organic compounds
}

\author{
Miquel Avella-Oliver, ${ }^{\mathrm{a}}$ Vicente Ferrando, ${ }^{\mathrm{b}}$ Juan A. Monsoriu, ${ }^{\mathrm{b}}$ Rosa Puchades, ${ }^{\mathrm{a}, \mathrm{c}}$ Angel

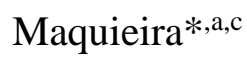 \\ a Instituto Interuniversitario de Investigación de Reconocimiento Molecular y Desarrollo \\ Tecnológico (IDM), Universitat Politècnica de València, Universitat de València, 46022 Valencia, \\ Spain. \\ ${ }^{\mathrm{b}}$ Centro de Tecnologías Físicas, Universitat Politècnica de València, 46022 Valencia, Spain. \\ c Departamento de Química, Universitat Politècnica de València, 46022 Valencia, Spain.
}

\begin{abstract}
Herein we present a diffractometric immunosensor to quantify low molecular weight organic compounds in a label-free, simple, and sensitive fashion. The approach is based on patterning analyte analogues (haptens) on solid surfaces according to a diffractive structure, and then loading specific antibodies on them to be subsequently displaced by free analytes in solution. This displacement generates a measurable change in the diffractive response that enables to quantify the analyte concentration. In this study we address the fabrication, optimization, and assessment of these diffractive structures of biological probes and their application to the analysis of atrazine, an organic compound extensively used as pesticide. This immunosensor displays well-correlated dose-response curves that reach a detection limit of $1.1 \mathrm{ng} \mathrm{mL}^{-1}$ of atrazine in label-free conditions. From a general viewpoint, this study also aims to provide insights into exploiting this approach towards prospective in-field analysis and screening strategies to sense multiple low molecular weight compounds in label-free conditions.
\end{abstract}

Keywords: biograting, diffraction-based sensing, atrazine, label-free, microcontact printing, displacement immunoassay. 


\section{Introduction}

The convergence of molecular sensing with nanoscience and nanotechnology has given rise to a range of measurement techniques that use different nanoscale phenomena for biosensing $[1,2]$. These systems typically exhibit label-free capabilities that enhance their analytical scope, for instance by improving the reliability of the results and by simplifying the analytical procedure [3$5]$.

Herein we focus on diffraction-based sensing (DBS), an optical technique that exploits light diffraction to analyze biorecognition events. DBS is grounded on patterning onto solid supports biological probes (proteins, antibodies, nucleic acids, etc.) according to a grating structure designed to diffract an incident laser beam, as depicted in Fig. 1A-B. Then, target compounds interact with these gratings (typically by binding), thus changing the amount of matter that constitutes the grating, which modifies its diffraction efficiency and consequently the intensity of the diffracted orders changes too. As a result, molecular-scale events can be sensed by measuring the diffraction pattern, e.g. by monitoring the intensity of a diffracted beam (Fig. 1C).

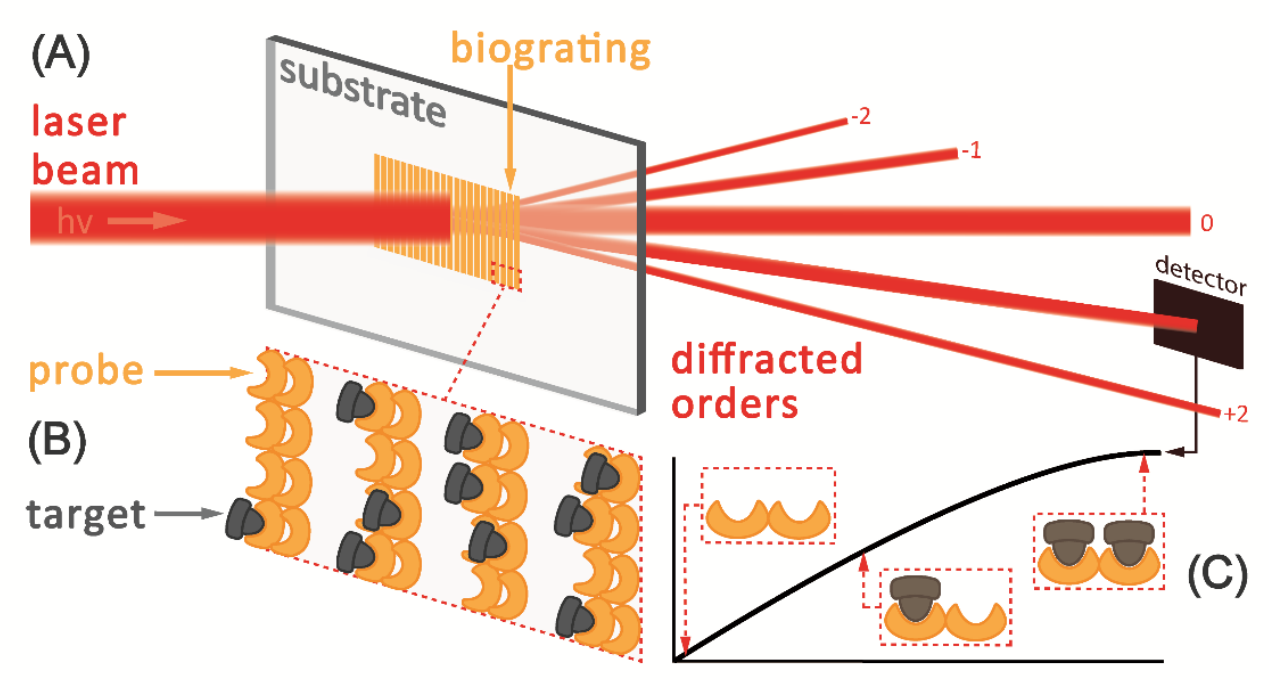

Fig. 1. Scheme of diffraction-based sensing at (A) macroscopic and (B) microscopic scale. (C) Exemplary real-time monitoring of a recognition event.

DBS and its bioanalytical implementation were firstly introduced in the scientific literature by Tsay and coworkers [6], then further explored mainly by the Goh's lab [7, 8] and other teams [9, 10], and more recently by Wang et al $[11,12]$. Axela Inc. introduced important advances in the field by commercializing its dotLab ${ }^{\mathrm{TM}}$ System, an integrated benchtop device for automatically running 
routine binding assays in a particular DBS configuration [13, 14]. Also, we recently developed a high-throughput system to analyze multiplexed endpoint DBS assays by exploiting the resources of compact disk technologies [15]. Along these lines, the concept behind diffractive biosensing is still expanding its scope by new approaches, such as focal molography [16].

Unlike most nanostructured bioanalytical systems [2, 4, 17, 18], DBS entails optically active structures constituted by the bioreceptors themselves patterned on plain solid substrates (Fig. 1B), without further pre-patterned supports. Herein we employ the term "biograting" to denote these biomolecular networks. Biogratings allow for conceiving transduction systems for label-free and real-time analysis in which the analytical signal only depends on the state of the bioreceptor, but not on the structuration of the substrate. Also, this kind of diffractive bioreceptor structures entails the unique strength of being potentially insensitive to the effects of non-specific binding [19].

In this study we combine the strengths of biogratings and displacement immunoassays to develop, for the first time, a DBS immunosensor to determine low molecular weight organic compounds. The approach is based on loading specific antibodies in analyte analogues (haptens) patterned on a surface, to be subsequently displaced by free analytes in solution. Unlike other competitive systems, in displacement immunoassays only the sample has to be handled (i.e. no further bioreagent additions are required) once the functionalized substrate is prepared, thus involving a simpler analytical procedure for final users. In addition, this assay format presents a preconcentration-like effect, in which the more sample interact with the system, the more antibody is displaced and the higher sensitivity is obtained.

Promising attempts to quantify low molecular weight organic compounds have been previously reported using diffractive gratings constituted by stimuli-responsive hydrogels (for glucose sensing) $[20,21]$ and molecularly imprinted polymers (for 2,4-D and enrofloxacin analysis) [12, 22]. These approaches provide good solutions for particular applications, but present rather moderate sensitivity (estimated detection limits from 14 to $60 \mu \mathrm{M}$ ) and selectivity (especially for hydrogels).

Herein, we present a sensitive DBS label-free biosensor for atrazine based on a displacement immunoassay. Atrazine is one of the most used pesticides in the world, banned in Europe from 2004 and widely employed in many other areas nowadays, especially in the US [23-25]. This is a highly persistent contaminant in water, atmosphere, and soil, and entails important human's health and ecotoxicological implications [25-27]. In addition to prove the concept for label-free DBS quantification of low molecular weight organic compounds, this work also aims to provide insights into the potential of this approach for simple in-field analysis. 


\section{Experimental section}

\subsection{Materials}

Buffer solutions employed in this study, sodium phosphate buffer (PBS, $8 \mathrm{mM} \mathrm{Na} 2 \mathrm{HPO}_{4}, 2 \mathrm{mM}$ $\mathrm{KH}_{2} \mathrm{PO}_{4}, 137 \mathrm{mM} \mathrm{NaCl}, 2.7 \mathrm{mM} \mathrm{KCl}, \mathrm{pH}$ 7.4), PBS-T (PBS with polysorbate $200.05 \%$ v/v), PBStau (PBS containing taurine 0.1 M), and PBS-TT (PBS-tau with polysorbate $200.05 \% \mathrm{v} / \mathrm{v}$ ), were prepared with purified water (Milli-Q, Millipore Iberica, Madrid, Spain). Polydimethylsiloxane (PDMS) Sylgard 184 was from Dow Corning (Wiesbaden, Germany). Bovine serum albumin (BSA), gelatin, hemoglobin, polysorbate 20 (Tween 20), taurine, and anti-BSA rabbit IgGs were supplied by Sigma-Aldrich (Madrid, Spain). Atrazine, carbaryl, simazine, and irgarol 1054 were from Riedel-de Haën (Seelze-Hannover, Germany). Chlorpyrifos was provided by Laboratorios Alcotan (Dos Hermanas, Spain), and casein was from Merck (Madrid, Spain). Glass slides were purchased from Labbox (Mataró, Spain). Atrazine protein-hapten conjugates were synthetized and anti-atrazine antibodies (polyclonal rabbit antisera) were obtained by immunization, as described elsewhere [28].

\subsection{Patterning}

In this study, protein biogratings were created on glass substrates by microcontact printing $(\mu \mathrm{CP})$. For that, polymeric diffractive lenses containing a binary grating of parallel lines (Fig. S1A) were used as masters to create the stamps for $\mu \mathrm{CP}$. PDMS was poured on the grating side of the master, degassed $30 \mathrm{~min}$ by vacuum, and polymerized overnight at $60{ }^{\circ} \mathrm{C}$. PDMS stamps were peeled off and cut in $4 \times 4 \mathrm{~mm}$ squared pieces. Prior to their use, stamps and substrates were sonicated $5 \mathrm{~min}$ in ethanol (30\% in water) and dried under a stream of air.

$50 \mu \mathrm{L}$ of probe solutions (BSA in sections 3.1-3.2 and BSA-hapten in sections 3.3-3.5) in PBS were placed on the grating side of the stamps for inking during a given time (inking time). Afterwards, stamps were rinsed with water and dried under a stream of air. Then, the grating side of the stamps was placed on the substrate surface, slight pressure was initially applied on the opposite side to favour the contact, and probes were let to transfer during a given time (stamping time) in order to immobilize on the glass surface by physisorption. Finally, stamps were removed and the resulting biogratings were rinsed with PBS-T and water, and dried as before. After using them, the stamps were washed 3 times by sonication ( $5 \mathrm{~min}$, ethanol $30 \%$ in water) and stored in this ethanolic solution to be reused. 


\subsection{Characterization}

Biogratings were characterized by atomic force microscopy (AFM), by noncontact mode in air using a Multimode 8 microscope (Bruker) and RFESPA probes (MPP-21120-10, Bruker). Nanoscope software was used for the AFM analysis, and first order polynomial flatten was applied to the data. Averaged cross section profiles were obtained by averaging, within a given area, every row of data along the longitudinal direction of the biograting strips.

\subsection{Detection setups}

The experimental configuration for diffraction-based sensing must enable light to efficiently interact with the biogratings and to project an output diffraction pattern that favor the measurement of the diffracted orders. Two different arrangements were used in this study: transmission and total internal reflection (TIR). In both cases, the measurements were experimentally performed with custom optomechanical setups (Fig. S2), using a collimated $650 \mathrm{~nm}$ diode laser as light source (LDM650/5LJ, Roithner Lasertechnik GmbH, Vienna, Austria) and employing a monochromatic CMOS camera as detector (Edmund eo-1312m, York, UK) to monitor the intensity of the fifth diffraction order to be used as analytical signal (Fig. S1).

In the transmission configuration, the substrate was positioned between the light source and the detector. Therefore, the laser beam goes through the substrate, interacts with the biograting, and the outgoing diffracted pattern can be monitored (Fig. 2A and Fig. S2A). According to experimental results and Fresnel conditions, the reflection/transmission intensities ratio in this detection setup greatly depends on the incidence angle of the laser beam (Fig. S3). Therefore, orthogonal laser incidence with respect to the substrate surface was selected in the transmission measurements for maximal sensitivity.

In the TIR setup, the laser beam hit the substrate at an incident angle that meets the total internal reflection condition at the interface that contains the biograting. Therefore, light interacts with the grating by means of evanescent waves, the diffraction pattern is reflected, and its intensity can be monitored (Fig. 2B and Fig. S2B). Considering Snell's law, experimentally impracticable angles (above 90 degrees) to reach the TIR condition should be applied when having air or aqueous solutions in the interface that contains the biograting. Therefore, the irradiation was mediated by a prism coupled to the glass substrate, and glicerol was added between both elements as index matching agent. Custom gaskets were attached on the substrates in order to incubate solutions on the biogratings (Fig. S2B).

In this study, the transmission setup was always used for endpoint measurements in dry conditions, and the TIR configuration always employed for real-time measurements in wet conditions (i.e., 
with aqueous solutions on the biogratings). Transmission leads to higher signal-to-noise ratios (Fig. 2C), but the exponential decay of the evanescent field in TIR confines light at the interface that contains the grating and restricts its interaction with the bulk of the sample [8]. Therefore, even if samples are kept on the biograting during the measurements, the effect of potential interferences from their interaction with the laser (absorption, scattering, etc.) is minimal, which makes this configuration particularly suitable for real-time measurements.
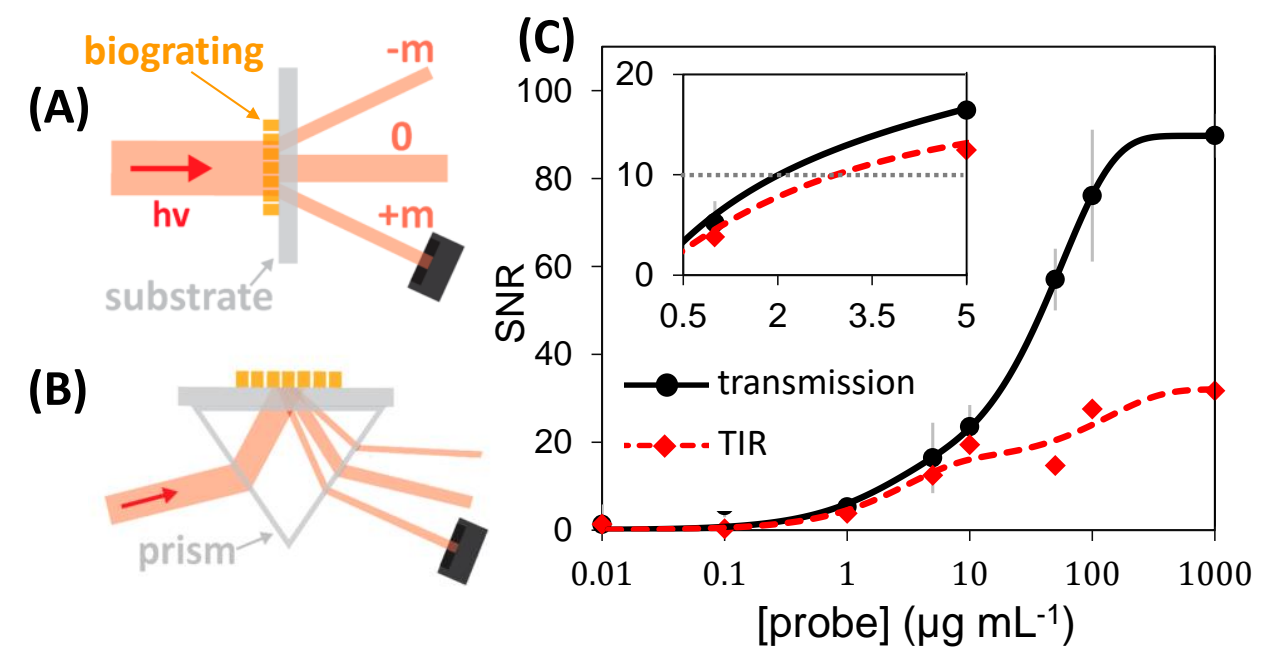

Fig. 2. Schemes of the detection setups for (A) transmission and (B) TIR. See Fig. S2 for real images of the setups. (C) Experimental results of protein adsorption isotherms measured by transmission and TIR. The standard deviations of ten blank gratings $\left(0 \mu \mathrm{g} \mathrm{mL}^{-1}\right.$ of probe) were used as noise values to calculate signal-to-noise ratios (SNR) and the graph inset zooms in on the quantification limit area $(\mathrm{SNR}=10)$. Experimental data fit to exponential equations (rise to maximum 4 parameter, $R_{\text {trans. }}^{2}=0.996$ and $\left.R_{T I R}^{2}=0.947\right)$.

\subsection{Assays}

To perform dose-response curves, IgGs and atrazine solutions $(50 \mu \mathrm{L})$ were incubated on the patterned biogratings at room temperature (about $22^{\circ} \mathrm{C}$ ) in a humidified environment (to prevent evaporation). For endpoint measurements, the biogratings were rinsed with PBS-T and water, and dried under a stream of air after each incubation. In real-time analysis, IgGs and atrazine solutions were added on small and known volumes of buffer that were previously dispensed on the biograting and let to stabilize for $10 \mathrm{~min}$ in order to maintain the hydration state of the patterned probes. 
A biorecognition system based on BSA as probe and anti-BSA IgG as target was used for the biogratings fabrication and assessment (sections 3.1-3.2). This is a useful model system for the subsequent displacement immunosensor, while also expands the scope of this assessment thanks to its high representativeness in the biosensing field. PBS-tau was used to improve the IgGs stability [29], and concentrations of $1000 \mu \mathrm{g} \mathrm{mL}^{-1}$ for proteins and $0.05 \%$ (v/v) for polysorbate 20 in PBS-tau were used for blocking.

For the atrazine displacement immunosensor (section 3.3-3.5), biogratings of protein-hapten conjugates were created on glass as described above, and selective IgG (1/50 dilution factor) and atrazine solutions in PBS-TT were incubated. This IgG presents high on-rate constants for the protein-hapten selected in this study (BSA-2a) and the free analyte $\left(1.6 \cdot 10^{9}\right.$ and $4.1 \cdot 10^{6} \mathrm{~s}^{-1} \mathrm{M}^{-1}$, respectively) and negligible off-rate constants for them, as quantified elsewhere [30]. The detection limits of this system were calculated as the analyte concentration associated to a $10 \%$ displacement, inferred from the sigmoidal regression of experimental dose-response curves [31].

\section{Results and discussion}

\subsection{Biogratings development and characterization}

Microcontact printing $(\mu \mathrm{CP})$ was herein employed as a patterning technique to create protein biogratings. This is an appealing approach for this purpose [32, 33], and its simplicity, inexpensiveness, and versatility offset its moderate reproducibility [34]. Furthermore, once obtained the masters, countless biogratings can be easily produced without requiring nanofabrication facilities. Herein, protein probes were patterned on glass substrates by $\mu \mathrm{CP}$ according to a grating structure that distribute diffraction efficiencies mainly in 3 orders (Fig. S1). In this section, the behavior of these biogratings was studied through protein adsorption isotherms. Three key variables were addressed (inking time, stamping time, and protein concentration in the inking solution) using a biorecognition model system based on BSA as probe and anti-BSA rabbit IgGs as target.

First, the diffraction intensity of protein gratings obtained by different inking times was measured to investigate this variable (Fig. 3A). Considering preliminary results (Fig. S4), experimental conditions to ensure high protein transfer were selected. The kinetics of inking is hindered by the moderate affinity of the protein for the elastomer used for the stamps (PDMS). This intermediate affinity must be high enough to enable probe adsorption from solution during inking, and lower than the one of the substrate to ensure the subsequent protein transfer. Then, stamping was studied by the same strategy. The stamping kinetics is favored by the higher protein affinity for the substrate and hindered by the solid-state nature of this process, but behaves much faster than inking 
(Fig. 3B). Finally, the probe concentration in the inking solution was also studied as before, which displayed the expected response for a protein adsorption isotherm (Fig. 3C). From all these results, an inking time of $160 \mathrm{~min}, 20 \mathrm{~min}$ for stamping time, and a protein concentration in the inking solution of $250 \mu \mathrm{g} \mathrm{mL} \mathrm{mL}^{-1}$ were selected as optimal biograting fabrication conditions for the subsequent experiments.

Surface mappings of biogratings from different protein concentrations were studied by AFM. Although flatter substrate surfaces at the nanoscale would favor this topographic characterization, consistent results were obtained by averaged cross section profiles (Fig. S6). As shown in Fig. 3D, the topographic measurements clearly reveal the biogratings' structure. The dependence between grating height and protein concentration fitted an exponential curve that reached a maximal height of $3.5 \mathrm{~nm}$ at $1000 \mu \mathrm{g} \mathrm{mL}-1$ of BSA (Fig. S7).

Only a small proportion of the proteins in the inking solution becomes finally patterned by $\mu \mathrm{CP}$ [35]. In this study, we experimentally observed that optimal biograting fabrications consumed negligible BSA concentrations. Therefore, the practical efficiency of this approach can be easily improved by just reusing the inking solutions.

(A)

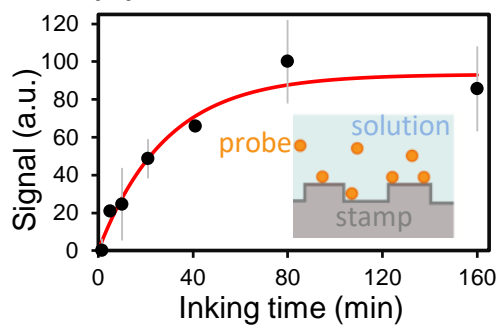

(B)

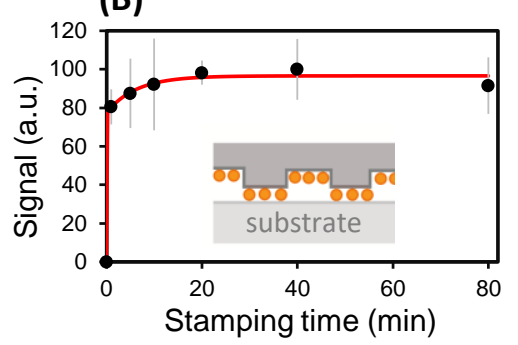

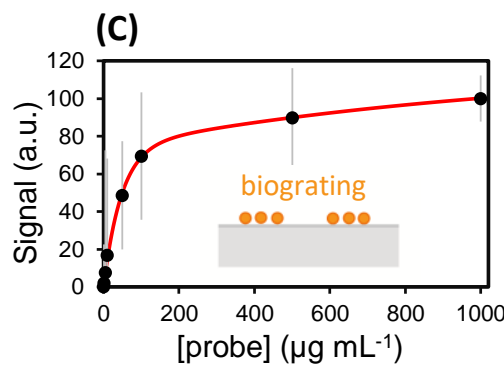

[probe] $\left(\mu \mathrm{g} \mathrm{mL} \mathrm{m}^{-1}\right)$

(D)
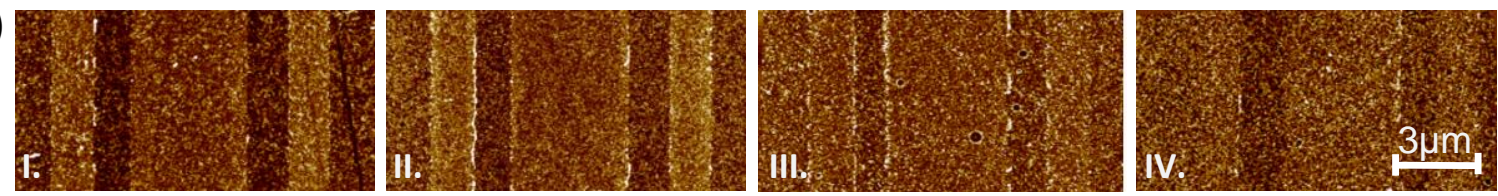

Fig. 3. Experimental assessment of the biograting fabrication variables: (A) inking time ([probe] $=$ $\left.100 \mu \mathrm{g} \mathrm{mL}^{-1}, \mathrm{t}_{\text {stamping }}=60 \mathrm{~min}\right)$, (B) stamping time $\left([\right.$ probe $\left.]=100 \mu \mathrm{g} \mathrm{mL}^{-1}, \mathrm{t}_{\text {inking }}=160 \mathrm{~min}\right)$, and (C) probe concentration ( $t_{\text {inking }}=160 \mathrm{~min}$, $t_{\text {stamping }}=25 \mathrm{~min}$ ). Data obtained by transmission measurements (see Fig. S5 for TIR results) and experimental curves fitted to an exponential (rise to maximum, 5 parameters) equation $\left(R_{\text {ink. }}^{2}=0.963, R_{\text {stamp. }}^{2}=0.994\right.$, and $\left.R_{[\text {prot }]}^{2}=0.999\right)$. Inset images are schematic illustrations of the corresponding $\mu \mathrm{CP}$ stages at microscopic scale. Normalized signal in the vertical axes. (D) AFM data of probe gratings using (I) 1000, (II) 100, (III) 10 , and (IV) $1 \mu \mathrm{g} \mathrm{mL}^{-1}$ of probe protein in the inking solution. 


\subsection{Blocking and stabilization}

The diffraction response strongly depends on the state of the interface that contains the biograting. Modifications (e.g., solution additions and blocking events) have major effects on the system. This section characterizes the nature of these processes in order to understand and control them (using the BSA/anti-BSA model system).

By real-time measurements, it can be observed that adding aqueous solutions to dry protein gratings causes an abrupt signal decay followed by an exponential stabilization (Fig. S8). This phenomenon can be explained by the greater refractive index $(\mathrm{RI})$ difference between proteins and air, compared to proteins and water $\left(\mathrm{RI}_{\text {proteins }} \approx 1.4, \mathrm{RI}_{\text {air }} \approx 1, \mathrm{RI}_{\text {water }} \approx 1.3\right)$. Therefore, adding aqueous solutions reduce the RI contrast between protein and gap strips in the biogratings, which decreases diffraction efficiency. Besides, the substrate surface and the immobilized probes may be susceptible to solvation, which might lead to significant conformational changes and affect the diffracted efficiency too. In the model system under study, $10 \mathrm{~min}$ solvation ensures enough stability for subsequent steps (Fig. S8).

Once stabilized, biogratings must be blocked (backfilled) to prevent non-specific adsorptions. Blocking agents in DBS bind available active sites of the substrate surface, mainly in biograting gaps, but also in probe areas. Different proteins (hemoglobin, BSA, gelatin, and casein) and a surfactant (polysorbate 20) were studied as blocking agents. Firstly, it was experimentally demonstrated that blocking allows to achieve this system's maximal performance. As shown in Fig. 4A, more than 2- and 5-fold signal increases were obtained when performing recognition after blocking with hemoglobin and polysorbate 20 , respectively.

At microscopic scale, it was observed that the relative height of the probe strips compared to gaps was much shorter for blocked biogratings (Fig. 4B), which indicates selective adsorption of blocking agents in the gaps. After target recognition, height increased in blocked biogratings but decreased in non-blocked conditions. This fact points out significant target adsorptions on unblocked gaps and selective target binding in the probe strips of blocked biogratings.

The behavior of the blocking agents in this system was also studied by real-time measurements. As shown in Fig. 4C, adding blocking solutions initially lead to sharpened diffraction efficiency decreases. This effect could be related with the lower RI contrast when blocking agents adsorb in the biograting gaps, and also with the higher RI of the solutions that contain blocking agents compared with plain buffer.

Different trends were observed after this initial sharpened decay (Fig. 4C). BSA and gelatin kept a stable signal level, while hemoglobin and casein initiated an exponential diffraction efficiency 
raise. Diverse processes related with the nature of the proteins in interfaces might be involved in this non-systematic phenomenon (adsorption kinetics, aggregation, solvation, conformational changes, etc.). Polysorbate 20 described a simple signal diminution, which we attribute to its rapid adsorption and stabilization at the interface.

From these results, polysorbate 20 was selected as the blocking agent for the following studies. Moreover, it was experimentally observed that individual blocking agent incubations can be skipped by including this surfactant in the target matrix (Fig. S10).
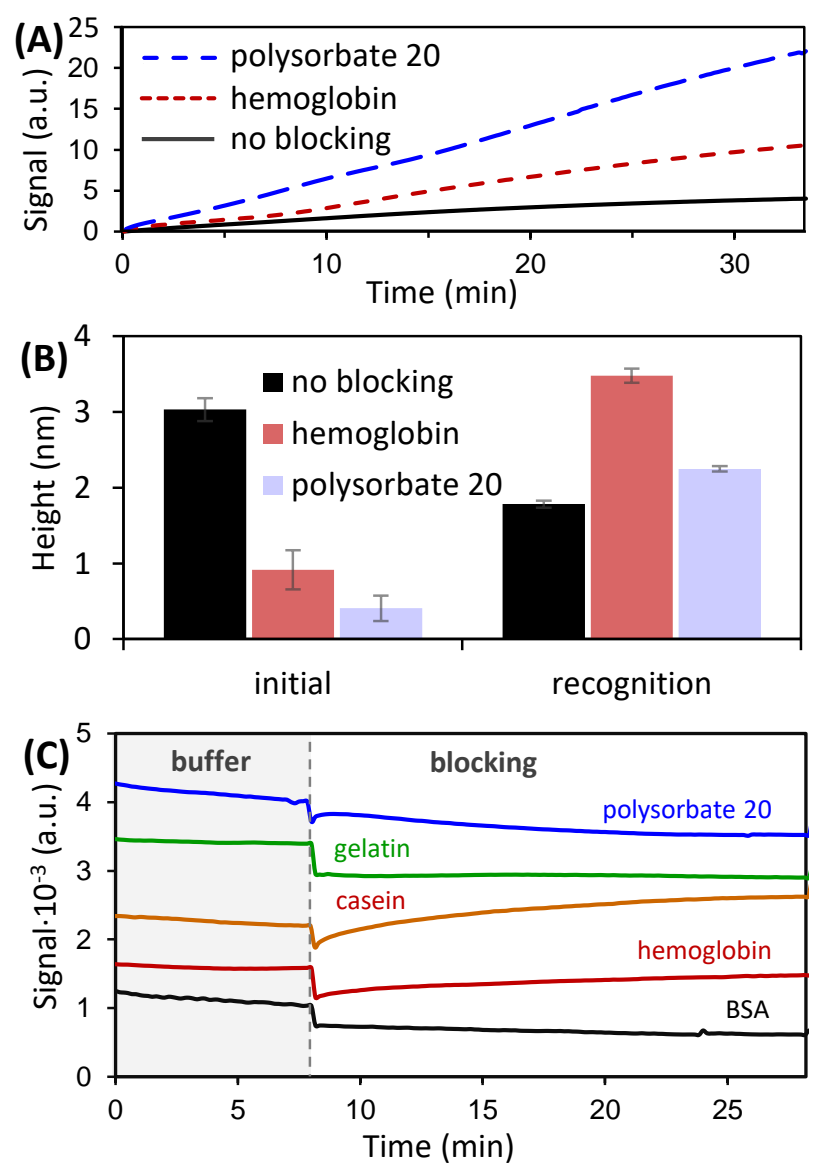

Fig. 4. Blocking and stabilization results. (A) Real-time recognition curves with and without blocking agents. (B) Grating height displayed by AFM (Fig. S9) for the studied blocking and target recognition. (C) Real-time curves for stabilization (buffer) and blocking stages, using different blocking agents. 


\subsection{Bioreagents assessment}

This section addresses an experimental screening study for a set of atrazine bioreagents. It must be noted that the recognition of low molecular weight compounds (such as atrazine) by immobilized antibodies leads to rather negligible changes in the amount of matter on the sensing surface. Also, small organic compounds can not simultaneously bind two antibodies, so sandwich immunoassays are not suitable and competitive formats must be implemented.

To develop a DBS displacement immunoassay to sense atrazine, herein biogratings constituted by BSA-hapten conjugates (Fig. 5A i.) were fabricated according to the optimal conditions previously reported in this work, since probe adsorption is mediated by BSA in both cases. Then, antibodies against atrazine were incubated on the biogratings to bind the immobilized haptens until saturation (Fig. 5A ii.). Once antibodies are loaded, free atrazine in samples competes with immobilized haptens for the binding sites of the antibodies, thus displacing them from the biogratings to the solution (Fig. 5A iii.). Therefore, atrazine additions lead to significant reductions in the matter constituting the biogratings (Fig. 5A iv.), which is transduced as a diffraction efficiency decrease.

In displacement immunoassays, the affinity of antibodies for immobilized haptens must be good enough to enable high antibody loading, and also high for the analyte in order to generate great displacements that favor sensitive analysis. Considering the experimental results of this bioreagents screening (Table S1), the conjugate BSA-2a and the antibody KLH-2d (IV) were selected, since this combination led to the highest displacement for atrazine additions.

As shown in Fig. 5B (stages 1-3), the expected diffractive signal variations were experimentally obtained along the different stages of this displacement assay. It can be observed that blocked BSA2a biogratings generate a low diffracted intensity, which increases about two orders of magnitude after loading the specific antibody, and decreases after adding free atrazine in solution.

\subsection{Biogratings regeneration}

The regeneration of biogratings to be used in more than one analysis was herein studied. Thought the presented approach meets the concept behind disposable chips for single assays, regenerating the biogratings could offer interesting options in terms of green and costs issues. Since the analysis procedure does not modify the protein-hapten biograting (but just displaces antibodies bound on it) this regeneration only requires a subsequent antibody incubation in order to reload them on the biograting.

To study this issue, successive antibody loads and analyte incubations were performed and their endpoint response was analyzed by transmission. As shown in Fig. 5B, the sensing capabilities of these biogratings can be only partially regenerated through this approach. Two different trends are 
observed in these results. First, after the initial analysis cycle, the signal of loaded biogratings underwent a significant decrease and remained constant in successive cycles. This fact could be related with different phenomena taking place during incubations and washing steps, such as desorption of the stamped probes, instability of the BSA-2a conjugates, and loss of activity by conformational changes of the immobilized probe. Future studies focused on the biograting regeneration should assess other protein-hapten conjugates and more effective probe immobilizations.

Second, it is also observed that the signal decrease caused by the displacement becomes smaller along the successive analysis cycles and tend to be null. This phenomena can be explained by the fact that polyclonal antibodies were used in this study, which contain a mixture of antibodies with different binding sites, some ones with higher affinity for free atrazine and others for the immobilized hapten. Therefore, the displacement of this second kind of antibodies during analyte incubations is not favored, thus becoming their population on the biograting increased along successive analysis cycles and causing a loss of sensitivity. Therefore, better regeneration capabilities could be obtained, for instance, by using monoclonal antibodies.

\subsection{Analytical performance}

To obtain the dose-response curve that characterizes the system, first the kinetic behavior of the assay was studied by real-time measurements. As observed in Fig. S11 A, the IgG loading on the biograting involves rather slow kinetics. From these results, overnight incubations (16 hours) were selected to load the antibody on the biograting, which corresponds to a $91.4 \%$ of its equilibrium state, according to the regression equation.

The antibody displacement by free atrazine in solution also entails slow kinetics (Fig. S11 B). It must be took into account that diffusion is the unique mass transport phenomena comprised in this system, so these values represent upper limits for the kinetics. Obviously, faster responses would be achieved by just introducing convection in the incubations (stirring, flow, etc.). As an alternative, we selected a kinetic method to drastically improve the assay times by using the slope of real-time displacement curves as analytical signal. As shown in Fig. 5C, the experimental kinetic dose-response curve for atrazine determination presents good results with one-minute incubations. A detection limit of $80.1 \mathrm{ng} \mathrm{mL}^{-1}(371 \mathrm{nM})$ was calculated from this curve, and longer incubation times did not improve this value.

Displacement dose-response curves were also performed by endpoint measurements. For that, atrazine solutions were incubated for $90 \mathrm{~min}$ on loaded biogratings, which corresponds to a $64.4 \%$ of the total biointeraction (Fig. S11). As shown in Fig. 5C, experimental data fits well to a sigmoidal 
curve and reach a detection limit of $1.1 \mathrm{ng} \mathrm{mL}^{-1}(5.1 \mathrm{nM})$, which is below the concentration limits stablished in the WHO's guidelines and EPA's regulations for atrazine in drinking water [36, 37].

(A)
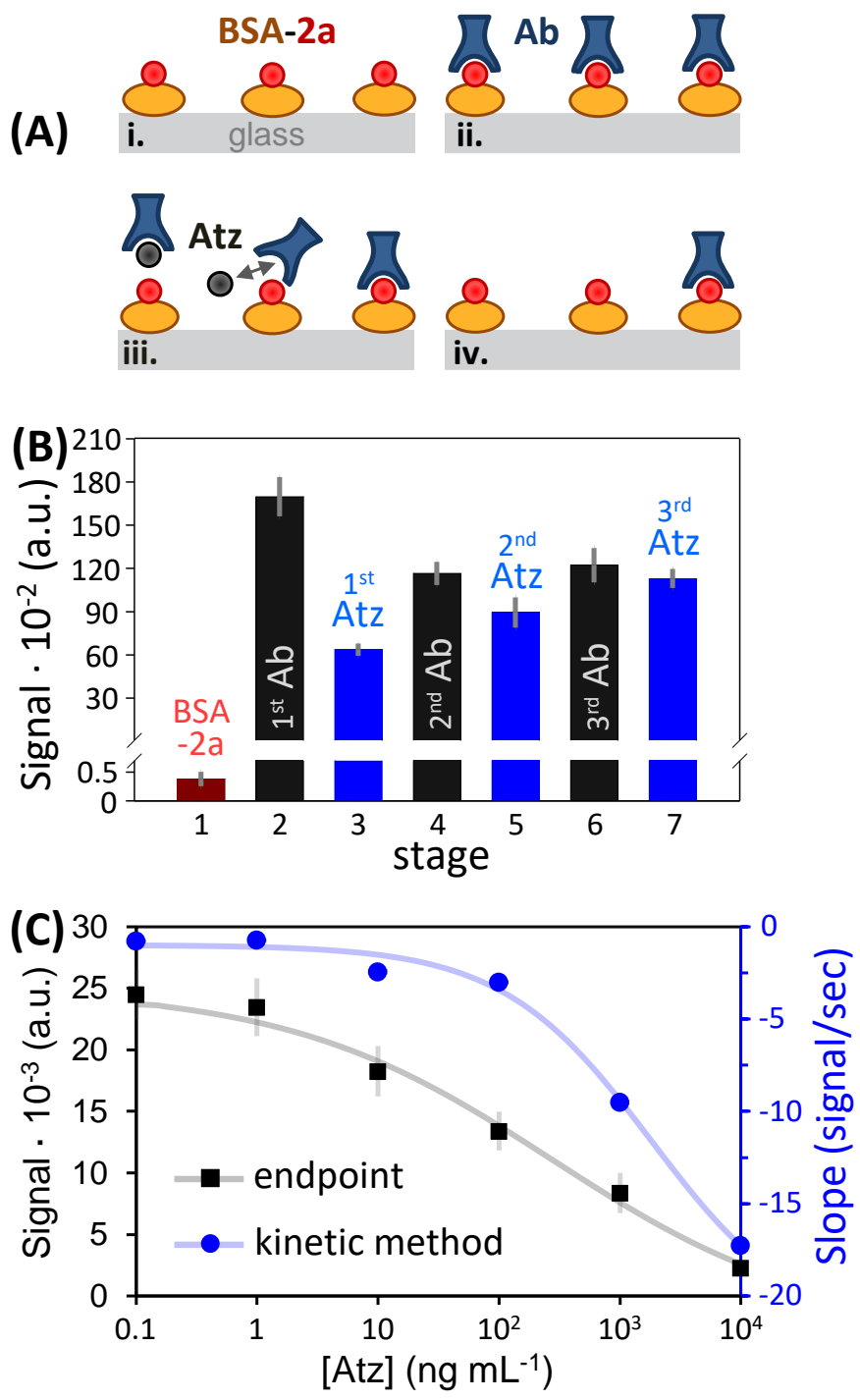

Fig. 5. Atrazine (Atz) displacement immunoassay. (A) Reaction scheme, including (i.) patterned probes, (ii.) antibodies ( $\mathrm{Ab}$ ) loading, (iii.) displacement, and (iv.) the resulting biograting after the assay. (B) Biograting regeneration results, comprising its original state (BSA-2a) and successive antibodies and atrazine incubations. (C) Experimental data and sigmoidal regression (logistic 4 parameters) for dose-response curves obtained by kinetic methods and by endpoint measurements $\left(R_{\text {kinetic }}^{2}=0.9944\right.$ and $\left.R_{\text {endpoint }}^{2}=0.9917\right)$. 
The selectivity of the system was experimentally assessed by incubating high concentrations $\left(10^{3}\right.$ $\mathrm{ng} \mathrm{mL} \mathrm{m}^{-1}$ ) of other pesticides as potential interferents, and comparing the resulting response with the one after incubating atrazine in the same conditions. Simazine and irgarol 1054 displayed a $28 \%$ and $16 \%$ of the displacement caused by atrazine, respectively. This substantial cross reactivity match with the fact that these two compounds are also based on the same triazine structure as atrazine. Lower interference were presented by chlorpyrifos and carbaryl (2\% and 9\%, respectively), since they belong to another class of pesticides.

Moreover, to provide insights into the performance of the system in more complex matrixes, the response of apple juice at different dilution ratios (in PBS-TT) on loaded protein-hapten biogratings was investigated. Two phenomena were experimentally observed (Fig. S12), which limit the applicability of the current approach and point towards prospective investigations on alternative biograting configurations. On the one hand, these incubations lead to a signal increase along time. On the other, they decrease the background signal after the incubations. The magnitude of both phenomena increment with the sample concentration, and they might be originated by desorption and non-specific binding processes on the biograting. Along these lines, negligible signal variations was observed for incubations of diluted (10\% in PBS-TT) juice in the current biograting configuration.

The main analytical features of representative studies on the analysis of atrazine in the recent scientific literature are summarized in Table S2, in order to assess this approach within the current state-of-the-art. From this comparison, the proposed strategy can be considered as an interesting alternative that provides enough sensitivity for particular applications, and enables label-free and real-time analysis involving minimal sample treatment and simple setups.

\section{Conclusions}

The results herein presented demonstrate that marrying diffraction-based sensing and displacement immunoassays is a sensitive approach to quantify low molecular weight organic compounds in label-free fashion, by both endpoint and real-time measurements. Microcontact printing has shown to be an appealing technique to create functional biogratings of protein-hapten conjugates on which loading specific antibodies to be subsequently displaced by the free analyte in solution. Kinetic methods and endpoint measurements both enable well-correlated dose-response curves that reach detection limits of 80.1 and $1.1 \mathrm{ng} \mathrm{mL}^{-1}$ for atrazine, respectively. The results obtained in this work also point out prospective strategies to further improve the capabilities of this approach in terms of sensitivity, faster responses, biograting regeneration, and analysis of complex matrixes. For instance, assays and biograting preparations could be easily speeded up by implementing simple 
convective setups for the incubations. Also, a flow system for the displacement stage would potentially increase the sensitivity of the assay, since free analytes contained in large sample volumes could displace loaded antibodies in an additive fashion, thus presenting a preconcentration-like effect. Besides, this immunosensor represents an appealing option for prospective in-field assays, since once fabricated and loaded with antibody, a chip containing the biograting could be easily brought to the point of need, incubated with the sample, and analyzed

using a simple optical setup. Furthermore, these results also open up a window for quantifying other low molecular weight organic compounds beyond atrazine, for instance to perform multiplexed label-free analysis of drugs, metabolites, pollutants, and biomarkers.

\section{Acknowledgements}

This work was supported by the Spanish Ministry of Economy and Competitiveness (CTQ201345875-R and FIS2011-23175), FEDER, and the Generalitat Valenciana (PROMETEO II/2014/040 and PROMETEO II/2014/072). Special thanks go to Richard A. McAloney and M. Cynthia Goh for hosting M.A.-O. as visiting researcher, sharing their expertise, and offering their valuable support. M.A.-O. also acknowledges the FPI program of the Spanish Ministry of Economy and Competitiveness for a $\mathrm{PhD}$ and an EEBB mobility grant.

\section{References}

[1] L. Rodriguez-Lorenzo, L. Fabris, R.A. Alvarez-Puebla, Multiplex optical sensing with surfaceenhanced Raman scattering: A critical review, Anal. Chim. Acta, 745 (2012) 10-23.

[2] M.C. Estevez, M.A. Otte, B. Sepulveda, L.M. Lechuga, Trends and challenges of refractometric nanoplasmonic biosensors: A review, Anal. Chim. Acta, 806 (2014) 55-73.

[3] R. Méjard, H.J. Griesser, B. Thierry, Optical biosensing for label-free cellular studies, Trends Anal. Chem., 53 (2014) 178-186.

[4] A. Kussrow, C.S. Enders, D.J. Bornhop, Interferometric Methods for Label-Free Molecular Interaction Studies, Anal. Chem., 84 (2012) 779-792.

[5] M.J. Banuls, R. Puchades, A. Maquieira, Chemical surface modifications for the development of silicon-based label-free integrated optical (IO) biosensors: A review, Anal. Chim. Acta, 777 (2013) 1-16.

[6] Y.G. Tsay, C.I. Lin, J. Lee, E.K. Gustafson, R. Appelqvist, P. Magginetti, R. Norton, N. Teng, D. Charlton, Optical biosensor assay (OBA), Clin. Chem., 37 (1991) 1502-1505.

[7] J.B. Goh, R.W. Loo, R.A. McAloney, M.C. Goh, Diffraction-based assay for detecting multiple analytes, Anal. Bioanal. Chem., 374 (2002) 54-56.

[8] J.B. Goh, R.W. Loo, M.C. Goh, Label-free monitoring of multiple biomolecular binding interactions in real-time with diffraction-based sensing, Sens. Actuators, B, 106 (2005) 243-248. [9] F. Yu, W. Knoll, Immunosensor with Self-Referencing Based on Surface Plasmon Diffraction, Anal. Chem., 76 (2004) 1971-1975. 
[10] G. Loget, R.M. Corn, Silica Nanowire Arrays for Diffraction-Based Bioaffinity Sensing, Chemistry (Weinheim an der Bergstrasse, Germany), 20 (2014) 10802-10810.

[11] X. Wang, X. Wang, Aptamer-functionalized hydrogel diffraction gratings for the human thrombin detection, Chem. Commun., 49 (2013) 5957-5959.

[12] X. Wang, X. Liu, X. Wang, Surface-relief-gratings based on molecularly imprinted polymer for 2,4-dichlorophenoxyacetic acid detection, Sens. Actuators, B, 220 (2015) 873-879.

[13] B.J. Pak, F. Vasquez-Camargo, E. Kalinichenko, P.L. Chiodini, T.B. Nutman, H.B. Tanowitz, I. McAuliffe, P. Wilkins, P.T. Smith, B.J. Ward, M.D. Libman, M. Ndao, Development of a Rapid Serological Assay for the Diagnosis of Strongyloidiasis Using a Novel Diffraction-Based Biosensor Technology, Plos Negl. Trop. Dis., 8 (2014).

[14] T.J. Gnanaprakasa, O.A. Oyarzabal, E.V. Olsen, V.A. Pedrosa, A.L. Simonian, Tethered DNA scaffolds on optical sensor platforms for detection of hipO gene from Campylobacter jejuni, Sens. Actuators B, 156 (2011) 304-311.

[15] M. Avella-Oliver, J. Carrascosa, R. Puchades, Á. Maquieira, Diffractive Protein Gratings as Optically Active Transducers for High-Throughput Label-free Immunosensing, Anal. Chem., 89 (2017) 9002-9008.

[16] V. Gatterdam, A. Frutiger, K.-P. Stengele, D. Heindl, T. Lübbers, J. Vörös, C. Fattinger, Focal molography is a new method for the in situ analysis of molecular interactions in biological samples, Nature Nanotechnology, advance online publication (2017).

[17] C.A. Barrios, V. Canalejas-Tejero, S. Herranz, M.C. Moreno-Bondi, M. Avella-Oliver, R. Puchades, A. Maquieira, Aluminum Nanohole Arrays Fabricated on Polycarbonate for Compact Disc-Based Label-Free Optical Biosensing, Plasmonics, 9 (2014) 645-649.

[18] M. Avella-Oliver, R. Puchades, S. Wachsmann-Hogiu, A. Maquieira, Label-free SERS analysis of proteins and exosomes with large-scale substrates from recordable compact disks, Sensors and Actuators B: Chemical, 252 (2017) 657-662.

[19] C. Fattinger, Focal Molography: Coherent Microscopic Detection of Biomolecular Interaction, Physical Review X, 4 (2014).

[20] G. Ye, X. Li, X. Wang, Diffraction grating of hydrogel functionalized with glucose oxidase for glucose detection, Chem. Commun., 46 (2010) 3872-3874.

[21] G. Ye, X. Wang, Glucose sensing through diffraction grating of hydrogel bearing phenylboronic acid groups, Biosens. Bioelectron., 26 (2010) 772-777.

[22] C.A. Barrios, C. Zhenhe, F. Navarro-Villoslada, D. López-Romero, M.C. Moreno-Bondi, Molecularly imprinted polymer diffraction grating as label-free optical bio(mimetic)sensor, Biosens. Bioelectron., 26 (2011) 2801-2804.

[23] Regulation (EU) NO 649/2012 of the European Parliament and of the council of 4 july 2012, Official Journal of the European Union, 27.7.2012.

[24] Edition of the Drinking Water Standards and Health Advisories, EPA, 2011.

[25] N.D. Jablonowski, A. Schäffer, P. Burauel, Still present after all these years: persistence plus potential toxicity raise questions about the use of atrazine, Environ. Sci. Pollut. Res., 18 (2011) 328-331.

[26] K.R. Solomon, J.A. Carr, L.H. Du Preez, J.P. Giesy, R.J. Kendall, E.E. Smith, G.J. Van Der Kraak, Effects of Atrazine on Fish, Amphibians, and Aquatic Reptiles: A Critical Review, Crit. Rev. Toxicol., 38 (2008) 721-772.

[27] N.D. Jablonowski, G. Hamacher, R. Martinazzo, U. Langen, S. Köppchen, D. Hofmann, P. Burauel, Metabolism and Persistence of Atrazine in Several Field Soils with Different Atrazine Application Histories, J. Agric. Food. Chem., 58 (2010) 12869-12877. 
[28] D. Jornet, M.Á. González-Martínez, Á. Maquieira, R. Puchades, Advanced Homogeneous-Heterogeneous Immunosensing Format Employing Restricted Access Supports, Anal. Chem., 79 (2007) 9331-9339.

[29] S. Damodaran, On the molecular mechanism of stabilization of proteins by cosolvents: role of Lifshitz electrodynamic forces, Langmuir, 28 (2012) 9475-9486.

[30] M. Avella-Oliver, D. Gimenez-Romero, S. Morais, M.A. Gonzalez-Martinez, P.R. Bueno, R. Puchades, A. Maquieira, INSEL: an in silico method for optimizing and exploring biorecognition assays, Chem. Commun., 49 (2013) 10868-10870.

[31] Immunoassay, in: E.P. Diamandis, T.K. Christopoulos (Eds.) Immunoassay, Academic Press, San Diego, 1996, pp. 569-579.

[32] C. Wendeln, B.J. Ravoo, Surface Patterning by Microcontact Chemistry, Langmuir, 28 (2012) $5527-5538$.

[33] J. Lee, K. Icoz, A. Roberts, A.D. Ellington, C.A. Savran, Diffractometric detection of proteins using microbead-based rolling circle amplification, Anal. Chem., 82 (2010) 197-202.

[34] V. Romanov, S.N. Davidoff, A.R. Miles, D.W. Grainger, B.K. Gale, B.D. Brooks, A critical comparison of protein microarray fabrication technologies, Analyst, 139 (2014) 1303-1326.

[35] A. Bernard, E. Delamarche, H. Schmid, B. Michel, H.R. Bosshard, H. Biebuyck, Printing Patterns of Proteins, Langmuir, 14 (1998) 2225-2229.

[36] Guidelines for drinking-water quality - 4th ed, World Health Organization2011.

[37] United States Environmental Protection Agency (October 2017) https://www.epa.gov/groundwater-and-drinking-water/national-primary-drinking-water-regulations. 